\title{
Pisando fuerte e invocando santos Formas corporales de conocimiento para caminar en el paisaje andino
}

\section{Cristina Fontes}

\author{
Universidad de Buenos Aires. Facultad de Filosofía y Letras. Instituto de Ciencias Antropológicas. Ciudad \\ Autónoma de Buenos Aires. Argentina \\ Correo electrónico: cristinafontes3@gmail.com \\ Magíster en Antropología Social (FLACSO). Investigadora asociada de la Sección Etnología del Instituto de \\ Ciencias Antropológicas, Grupo Culturalia. Facultad de Filosofía y Letras. Universidad de Buenos Aires
}

Recibido:

16 de noviembre de 2017

Aceptado:

o3 de julio de 2018

\section{Resumen}

Desde una perspectiva anclada en la antropología de la experiencia, este texto constituye una exploración etnográfica de la práctica de caminar en las actividades cotidianas de los pobladores de un sector rural de la Quebrada de Humahuaca, residentes de una comunidad local y trabajadores que, oriundos de la misma área geográfica, se desplazan en la zona por motivos laborales. Se analizarán diversos aspectos del conocimiento que utilizan para transitar por el paisaje a partir de las relaciones de intersubjetividad que establecen con los diferentes elementos que lo componen. La metodología empleada en esta investigación consistió en participar de los recorridos habituales que estos pobladores hacen en el transcurso de sus actividades cotidianas. Se realizaron, además, entrevistas abiertas con el fin de recoger datos sobre sus experiencias de caminar por ese paisaje.

\section{Stepping hard and invoking saints. Bodily ways of knowing to walk the Andean landscape}

\section{Abstract}

From a perspective based on the anthropology of experience, this paper is an ethnographic exploration of the practice of walking among inhabitants of a rural area of the Quebrada de Humahuaca (residents of a local community and workers native of the same geographical area, who move within it for work purposes) as part of their daily activities. It analyzes various aspects of the knowledge they resort to while traveling through the landscape, based on intersubjectivity relations that they establish with the different elements

\section{Key words}

Landscape; Corporality; Intersubjectivity; Walking; Southern Andes
Palabras clave

Paisaje; Corporalidad; Intersubjetividad; Práctica de caminar; Andes Meridionales 
within it. Research methodology consisted of taking part of the usual treks throughout the course of the day. In addition, open interviews were held in order to collect data about their experiences of walking the landscape.

\section{Pisando forte e invocando santos. Formas corporais de conhe- cimento para caminhar na paisagem andina}

\section{Resumo}

Palavras-chave

Corporeidade; Intersubjetividade; Prática de caminhar; Andes Meridionais
Adotando uma perspectiva ancorada na antropologia da experiência, este texto constitui uma exploração etnográfica da prática de caminhar entre os habitantes de um setor rural da Quebrada de Humahuaca como parte de suas atividades diárias. Analiso vários aspectos do conhecimento utilizado por seus habitantes para percorrer a paisagem a partir das relações de intersubjetividade que eles estabelecem com os diferentes elementos que a compõem. A metodologia utilizada nesta investigação consistiu em participar das rotas habituais que os habitantes de uma zona rural da Quebrada de Humahuaca (residentes de uma comunidade local e trabalhadores nativos da mesma área geográfica que caminham nela por razões laborais) fazem no curso de suas atividades diárias. Além disso, foram realizadas entrevistas abertas para coletar dados sobre suas experiências de caminhar por essa paisagem.

\section{Introducción}

La movilidad es un rasgo característico de los pobladores de la Quebrada de Humahuaca que está asociada a prácticas de la vida cotidiana, mecanismos de intercambio económico, traslados con fines ceremoniales y religiosos, desplazamientos vinculados a cambios en el clima y migraciones. Si bien el aumento de la disponibilidad de vehículos y del transporte público ha modificado los modos de moverse en el paisaje, muchos de los trayectos que forman parte de las actividades diarias se siguen haciendo a pie. Esto es particularmente cierto en aquellos pobladores que continúan haciendo de las actividades agropecuarias su modo de vida o que trabajan como agentes sanitarios o maestros rurales, ya que su única manera de llegar al trabajo, o de cumplir con él, es caminando.

Este texto constituye una exploración etnográfica de la práctica de caminar en la Quebrada de Humahuaca, con el objetivo de analizar diversos aspectos del conocimiento utilizado por sus pobladores para transitar por el paisaje. Para ello, se adoptará una perspectiva anclada en la antropología de la experiencia (Turner, 1982, 1985; Turner y Bruner, 1986; Jackson, 1989, 1996), entendiendo que la experiencia no estaría constituida solo por un conjunto de datos sensoriales o de conocimientos, sino que implica la existencia de un ser activo quien, además de participar en la acción, le da forma y reflexiona sobre ella. Se considerará a la acción de caminar como la práctica base de las actividades cotidianas y como una forma de adquirir un conjunto de conocimientos y de 
saberes localizados construidos a partir de las diferentes técnicas corporales empleadas en la marcha, la sensibilización del aparato perceptual del caminante y la recolección de información que se produce a lo largo de los recorridos que las personas realizan cotidianamente (Ingold, 2011). En ese sentido, el paisaje será definido como el mundo tal como es conocido y experimentado por aquellos que lo habitan mientras transitan los caminos y los lugares en donde desarrollan su vida diaria (Ingold, 2000).

En la literatura especializada en el área geográfica andina, la relación de los pueblos andinos con el paisaje fue tomada como objeto de estudio en numerosos trabajos que dieron cuenta de las particulares relaciones y construcciones sociales del espacio, ya sea en relación con actividades cotidianas o con prácticas ceremoniales y rituales ( $c f$. Martínez, 1969, 1989; Bastien, 1978; Isbell, 1978; Allen, 1981; van Kessel, 1992; Grillo Fernández, 1994; Abercrombie, 1998; Göbel, 2000-2002; Gil García y Fernández Juárez, 2008; Sendón, 2010; Cruz, 2012).

En el noroeste argentino, diversos autores han analizado la influencia del paisaje - en tanto espacio marcado por la sacralidad - sobre la salud y el bienestar de las personas (Bugallo, 2009; Vilca 2009; Bugallo y Vilca 2011) o de los animales (Bugallo 2016). Otros se han focalizado en la concepción y vivencia del espacio en el desarrollo de las actividades cotidianas de sus pobladores, tales como el pastoreo ( $c f$. Merlino y Rabey, 1983; Rabey y Merlino, 1988; Fernández García, Tecchi y Rabey 1992; Göbel 2002; Cladera 2010; Bugallo y Tomasi 2012; Tomasi 2013, 2014), las actividades agrícolas (Lema, 2014) o la movilidad vinculada a los intercambios comerciales (Karasik, 1984; Rabey, Merlino y González, 1986; Göbel, 1998; Nielsen, 2003; Abeledo, 2010; Bugallo y Mamaní, 2014). Por su parte, algunos investigadores, como Martínez (2014) y Lema y Pazzarelli (2015), se han dedicado a explorar la relación entre el paisaje, la movilidad y la memoria social de la comunidad.

Sin embargo, estas investigaciones se han centrado particularmente en el uso, interpretación y organización del espacio, y han dejado de lado el análisis de la experiencia y la práctica misma del caminar, por lo que el presente trabajo resulta un aporte original a los estudios de movilidad en esta zona.

La metodología empleada en esta investigación consistió en participar de los recorridos habituales que los pobladores de un área rural de la Quebrada de Humahuaca hacen en el transcurso de sus actividades cotidianas. El grupo de pobladores con los que se caminó y a los que también se entrevistó estuvo conformado por residentes de una comunidad local (Ocumazo) y por trabajadores (maestros y agentes sanitarios) que, oriundos de diversas localidades de la misma área geográfica, se desplazan en la zona por motivos laborales.

Se realizará una breve descripción del área en la que se desarrolló la presente investigación, y luego, de los modos en que los niños adquieren progresivamente el conocimiento indispensable para desplazarse en el paisaje. A continuación se analizarán las relaciones de intersubjetividad que el caminante establece, tanto con el suelo por el que transita, como con los diferentes seres tutelares cuya protección invoca antes de emprender el viaje o ante un eventual accidente durante la marcha. Finalmente, se examinarán las estrategias empleadas para enfrentarse al peligro que encierran las tormentas y los rayos como fenómenos meteorológicos particulares que forman parte de la experiencia del caminar en la región andina. 
1. El permiso para realizar esta investigación fue otorgado en el marco de una asamblea de la Comunidad Aborigen de Ocumazo en 2015. Los nombres de los interlocutores fueron cambiados para preservar su privacidad y anonimato, tal como prevé el consentimiento informado comunitario que ha sido firmado.

\section{Lugares y territorios: la comunidad de Ocumazo}

El área geográfica en la que se realizó el estudio está ubicada en el sector septentrional de la Quebrada de Humahuaca. Tomando la comunidad de Ocumazo como punto de referencia, dicha área quedó delimitada con base en la red de caminos que son o que han sido utilizados por los entrevistados para llegar a localidades vecinas en el desarrollo de sus actividades cotidianas (ver mapa).

Ocumazo $^{1}$ es una pequeña comunidad rural ubicada a $18 \mathrm{~km}$ al sudeste de la ciudad de Humahuaca (Jujuy). El poblado se encuentra en el fondo de un valle fértil atravesado por el río Calete, en cuyas márgenes se extienden pequeñas parcelas de cultivo. Para llegar a la comunidad es preciso cruzar los cerros que la rodean, ya sea en auto (por medio de sendas vehiculares de tierra y ripio) o a pie (por caminos de herradura y pequeños senderos que cruzan los cerros). Si bien el censo comunal registra unas 36 familias, la población estable no supera las 15. Al igual que ocurre en otras comunidades de la Quebrada, esto se debe a la gran movilidad de sus pobladores, la cual está vinculada a oportunidades laborales o educativas. Algunos de ellos incluso tienen dos casas: viven unos días de la semana en Humahuaca (donde además realizan otras actividades con las que contribuyen al ingreso familiar) y otros días en la comunidad, dependiendo del régimen escolar de los hijos o de los trabajos que haya que hacer en el campo. Otros pobladores han migrado y viven en lugares más alejados, como Mina El Aguilar, Palpalá, San Salvador de Jujuy o incluso Buenos Aires, pero mantienen el vínculo con la comunidad mediante la participación en las tareas agrícolas y asistiendo a las fiestas y celebraciones locales, en especial las realizadas en honor a la virgen tutelar del lugar, y en Carnaval.

La mayoría de los habitantes de Ocumazo son pequeños productores que cultivan sus parcelas en el fondo del valle bajo un sistema de riego por acequias. Al igual que ocurrió en otras zonas de la Quebrada (cf. Arzeno, 2011), las migraciones (muchas de ellas definitivas) de gran parte de la población en edad activa y su inserción al mercado laboral asalariado produjeron cambios

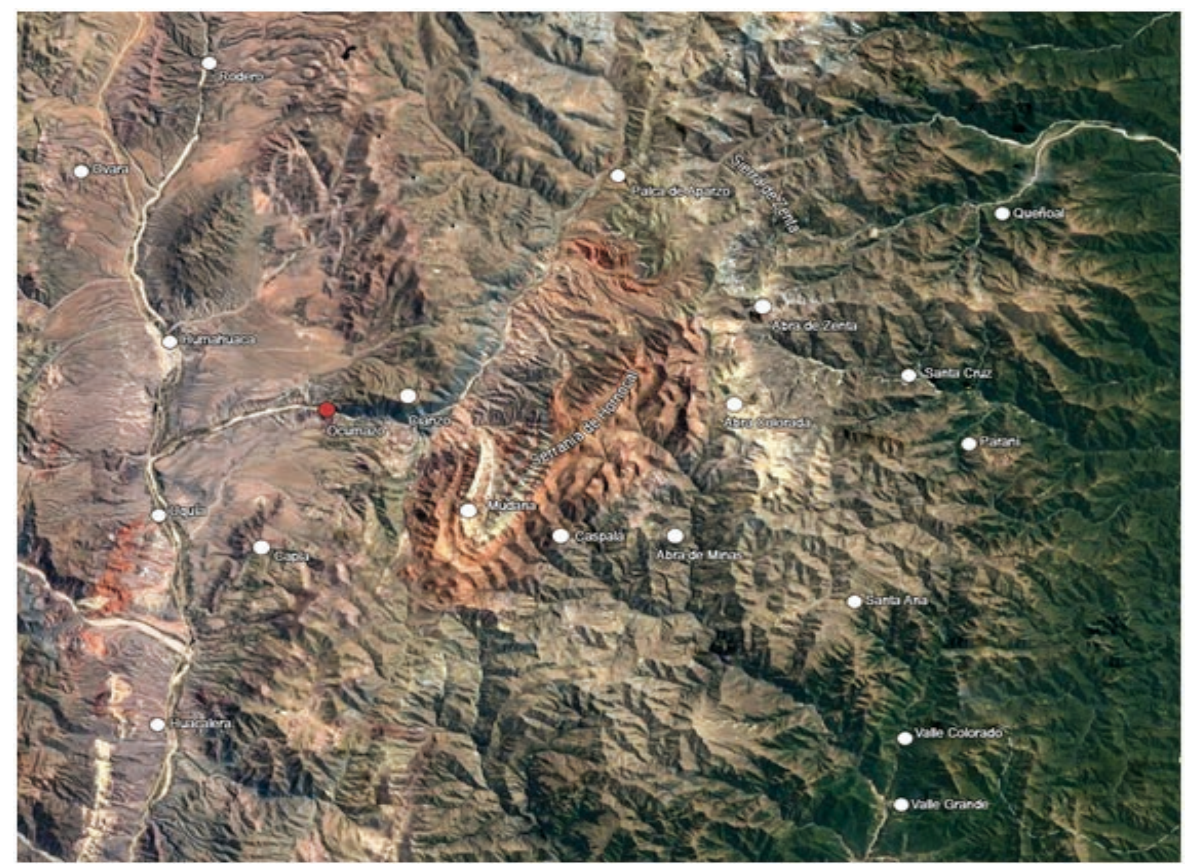


en las estrategias de vida y una disminución de las actividades agrarias en la comunidad. No obstante, existe un pequeño grupo de productores que residen en ella de modo permanente y que complementan sus ingresos provenientes de la producción agrícola con la cría de ovejas y de cabras, que llevan a pastorear a las laderas de los cerros que rodean el valle, al "campo" ${ }^{2}$ o a algún rastrojo dentro del mismo poblado. Algunos pocos también crían ganado vacuno, pero debido a que este requiere de una cantidad de forraje mucho mayor, lo hacen en terrenos ubicados en localidades vecinas. Esto los obliga a realizar periódicamente travesías que duran entre tres y cinco días para llegar a Caspalá, Santa Ana o Valle Grande - en la zona de los valles orientales - o viajes más cortos hasta Yala de Monte Carmelo, con el fin de revisar su hacienda. Aunque la mayor parte del trayecto lo suelen hacer a lomo de burro o a caballo, es frecuente que el mal estado de los caminos obligue a los viajeros a apearse de su montura y caminar en varias partes del recorrido.

Hasta hace unos tres años, los caminos que cruzan los cerros que rodean la comunidad eran la ruta obligada que debían tomar los maestros y algunos de los alumnos para llegar a la escuela, lo cual implicaba empezar a caminar antes del amanecer para poder cumplir con el horario de clases. Actualmente, tanto el personal docente como los alumnos se trasladan en un vehículo subvencionado por el Ministerio de Educación provincial que, de acuerdo con la disponibilidad de espacio, lleva también a algunos pobladores hasta Humahuaca. Pero cuando no hay lugar y es imperativo llegar a la ciudad, algunos caminantes continúan utilizando los antiguos senderos que, a través de los cerros, los conducen hasta los barrios ubicados en las afueras del centro urbano.

\section{Aprender el camino}

Tal como señala Ingold (2011), para todos los caminantes existe un trayecto a recorrer por primera vez del que solo saben el nombre del punto de partida y el de destino. Aunque el conocimiento general de la zona - ya sea obtenido en viajes anteriores o a partir de los relatos de terceros - le puede dar al viajero una idea aproximada acerca de la ubicación geográfica y de la distancia entre uno y otro lugar, el modo concreto de unir estos dos puntos debe ser aprendido en la práctica: cada caminante debe trazar su propio recorrido en el paisaje.

Para los pobladores de esta área rural, este aprendizaje comienza desde una edad muy temprana, mientras acompañan a sus padres o a sus hermanos mayores en la realización de las actividades cotidianas que hacen a la economía doméstica (el pastoreo de ovejas y cabras o la recolección de leña). En estos recorridos, van adquiriendo paulatinamente un conocimiento corporizado de los movimientos necesarios para transitar el paisaje que, a su vez, se articula con las habilidades, fórmulas y rutinas relacionadas con la subsistencia del grupo familiar. Al mismo tiempo que ejecutan estas tareas, los límites del espacio conocido se van extendiendo de manera progresiva a medida que se desarrolla la capacidad de marcha del niño: cuando solo tienen tres años, acompañan a sus hermanos mayores a "pastear" ovejas cerca de la casa; luego de los cinco, se considera que ya tienen edad suficiente como para ir con el rebaño al "campo". Las niñas suelen incluso quepear ${ }^{3}$ a algún hermanito que la madre dejó a su cargo mientras cuidan a los animales.

Si bien en diferentes estudios etnográficos (cf. Paradise y Rogoff, 2009; Gaskins y Paradise, 2010) todo este aprendizaje usualmente ha sido definido como "observacional", debido a la aparente centralidad del aspecto visual, en la
2. Espacios de menor altitud, abiertos y planos.
3. Quepear (del quechua k'epi) significa llevar una carga en la espalda. Es el modo habitual en que muchas mujeres cargan a sus hijos más pequeños. 
región andina los adultos esperan que los niños utilicen todos sus sentidos para aprender a desplazarse en el terreno, encontrar la huella ${ }^{4}$ y detectar eventuales peligros vinculados con las características del terreno o a la cercanía de animales silvestres (Pardo, 1997). En este sentido, se trata de un proceso de "educación de la atención" (Gibson, 1979 en Ingold, 2000), o de un aprendizaje basado en la percepción (Ingold, 2000) en el que el niño participa activamente, en determinadas condiciones del terreno y del paisaje, y relacionándose con otras personas expertas en la práctica. Es una forma de enseñanza diferente, por la cual los aprendices aprenden exponiéndose a una situación concreta bajo la guía y la tutela de alguien más experimentado, quien les muestra qué hacer y a qué estar atentos (Ingold, 2008). Tal como señala Pardo, en la crianza de los niños andinos no hay separación entre la teoría y la práctica: "el que sabe, lo sabe porque lo hace, y porque lo sabe, lo enseña" (Pardo, 1997:146). Este "saber" es, por lo tanto, el resultado de una experiencia vivida. Sin embargo, no se trata de un proceso totalmente "no verbal" (Paradise y Rogoff 2009: 117), ya que también se utiliza el lenguaje. Pero este está únicamente al servicio de la comunicación entre aprendiz y maestro con el fin de hacer observaciones y correcciones al niño durante la misma práctica y no para transmitir una serie de instrucciones abstractas sobre lo que debe hacer. En ese sentido, esta diferencia está más ligada al modo de conocimiento práctico propio de las sociedades orales o con una tradición oral todavía muy marcada (cf. Ong, 2006).

Luego de esta primera etapa en la que están acompañados de un experto, los niños van solos al campo o al cerro, donde ponen a prueba lo que han aprendido. Casi todos los entrevistados recordaron haber hecho ese primer recorrido con cierto temor. Juan, un maestro de Ocumazo, recuerda su propia experiencia:

Al principio tenés miedo: miedo de perderte o de que te aparezca una víbora... o cualquiera de esas cuestiones. Pero el papá siempre te dice que llevés un palo, que llevés la soga, que llevés eso y, bueno, entonces vos no estás desprotegido así nomás. Para eso ellos te llevan temprano, para que vos vayas adquiriendo todos esos conocimientos (Juan, 38 años, maestro).

Pero además, en los Andes se espera que los niños tengan una cierta cuota de iniciativa y de capacidad de resolución de problemas (Anderson, 2013). Si el niño no recuerda el camino, aprovecha el conocimiento del terreno que tienen los animales que lo acompañan. Debido a que estos suelen transitar siempre por un mismo sector del paisaje, conocen los senderos y los lugares donde el paso es más fácil, por lo que el niño solo tiene que seguirlos. Al mismo tiempo, se dedica a mejorar su conocimiento del área estableciendo referencias visuales a lo largo del camino: “En el campo nosotros teníamos los burros, y [los padres] te mandan con el burro, y el burro va por el sendero, iy ellos te llevan! Entonces, bueno, ¡ya está! Vas conociendo con él a la par y ya vas mirando" (Juan). De esta manera, el niño adquiere progresivamente y "sobre la marcha" ${ }^{5}$ (Ingold, 2000: 229) un conocimiento del paisaje local en cuya construcción intervienen no solo la observación y la experiencia de tránsito por una topografía cotidiana, sino también las relaciones de intersubjetividad que establece con los animales con los que convive estrechamente (cf. Elizaga, 2010; Bugallo y Tomasi, 2012).

Una vez que ganan confianza, los niños exploran el terreno para buscar nuevas rutas, y así ponen a prueba su destreza, su capacidad física y su habilidad de orientación en el paisaje. Por lo general, se trata de recorridos con una topografía difícil, con lugares de paso angostos o cubiertos con piedras sueltas que los obligan a ajustar sus movimientos con cada pisada para evitar caerse. De 
acuerdo con Ingold (2011), es en ese ajuste continuo del cuerpo en relación con el ambiente donde reside, en definitiva, la capacidad de caminar. Este aprendizaje individual es mantenido en secreto, y los niños toman precauciones especiales para no tener un accidente o no perder algún animal del rebaño: un percance de este tipo los delataría frente a sus padres y podrían ser castigados por no utilizar el camino que les fue enseñado: "si sabés que te has ido por ese lugar que tu papá no te enseñó y te has mandado una macana, no te quejes porque cobrás... iya!" (Juan). No obstante, se trata de instancias importantes en las que, mediante la aplicación de un sistema de prueba y error, los niños incrementan su conocimiento del paisaje y sus habilidades para desplazarse en él.

Pero para caminar en el paisaje andino no solo es preciso adquirir un conocimiento práctico, sino que también es necesario conocer ciertas características de los lugares por los que se transita y los gestos rituales que deben realizarse para evitar peligros durante el trayecto. Como ha sido señalado por diversos autores (cf. Mayer, 1974; Ortiz Rescaniere, 1989; Albó, 1991; van Kessel, 1992; Estermann, 2006), la cosmovisión andina se cristaliza en una organización social particular que se basa en el principio fundamental de la relacionalidad entre todos los seres y entre todos los acontecimientos. De acuerdo con este principio, el hombre nunca es un individuo autónomo, sino que está insertado dentro de un sistema de relaciones múltiples que le dan sentido y razón de ser, regulado por un conjunto de valores que son aprendidos y transmitidos generacionalmente en las prácticas de la vida cotidiana. Dicho sistema está centrado en la tierra - la Pachamama - , considerada como una madre universal e inmanente; cuidadora de la vida y de la salud; favorecedora de comerciantes y de viajeros; y protectora de los peligros. Sin embargo, pese a las asociaciones que la Iglesia católica ha querido establecer entre esta y la Virgen María, la Pacha es un ser tutelar ambiguo que, así como puede dar, también puede quitar si el hombre no respeta los deberes de reciprocidad hacia ella. De ello resulta una relación particular entre los pobladores con su medio natural que se manifiesta también en los modos de caminar por el paisaje.

Muy tempranamente, los niños aprenden que es preciso challar a la Pachamama antes de iniciar el camino y solicitar su permiso para recorrerlo con seguridad. Las challas consisten en una libación hecha con alcohol y hojas de coca que es acompañada de un respetuoso pedido de permiso para transitar por el lugar, sobre todo si se trata de uno que se va a recorrer por primera vez o si se debe caminar por lugares no habitados. A semejanza de lo descripto por Bugallo (2009) en la Puna y por Cruz (2012) en Bolivia, dichos lugares son ámbitos asociados con lo salvaje, fuera del espacio socializado y domesticado de la comunidad. Según Nancy, una agente sanitaria que trabaja en esta zona:

Son tierras vírgenes que no las usa nadie, nadie camina por ahí. Esas tierras, cuando vas, te "agarran", te "pillan”, te "tragan”, te hacen esas cosas. Entonces, vos siempre con permiso, porque estás en tierras que no son habitadas. Mis abuelos me enseñaron y, bueno, yo me quedé con esa enseñanza... (Nancy, 35 años, agente sanitaria).

Ser "agarrado" por la tierra se traduce físicamente en la aparición de granos y ronchas en todo el cuerpo, signos físicos que delatan la desobediencia del niño a las normas impartidas por sus mayores: "si vos después decías 'me fui al campo y mirá, tengo granos por todo el cuerpo', los abuelos te retaban: 'entonces te agarró la tierra, jte pilló! Porque vos andás como si nada, no pedís permiso..."' (Ariel, 30 años, residente de Ocumazo). 
6. Debido a que, en algunos lugares, esas apachetas han quedado situadas al lado de caminos vehiculares, su tamaño ha disminuido considerablemente, ya que son muy pocos quienes se detienen para cumplir con el ritual. Algunos ven esta "falta de respeto" a la tierra como la causa de los accidentes automovilísticos que ocurren en los caminos de montaña.

7. La peligrosidad de estos lugares se debe a que son considerados fenómenos sagrados de transición que comunican el orden humano con el orden cósmico, por emanar del vientre de la Madre Tierra.

8. Desgano o desánimo. Se debe a que el "ánimo" o principio vital que anima a los diferentes seres es "agarrado" por el lugar.

9. Algunos también hacen las tres cruces sobre la piedra.

10. Bola de hojas de coca que se masca para extraer su jugo estimulante.
Las challas se repiten también en las apachetas, montículos de piedra ubicados a la vera de los caminos en los cerros, en las abras o lugares de paso entre dos valles. Estas construcciones son el resultado de la acumulación de piedras depositadas por los viajeros al pasar por allí, por lo tanto, su tamaño es considerado como un indicador de la cantidad de personas que transitan el camino. ${ }^{6}$ En cada piedra que deja en la apacheta, el caminante entrega su cansancio a la tierra para poder continuar su marcha más aliviado.

Los niños también aprenden que deben evitar descansar en otros lugares considerados potencialmente peligrosos como las cercanías de los "ciénegos", lugares pantanosos cercanos a un ojo de agua, ya que son considerados lugares "bravos" 7 que pueden afectar la salud del viajero ocasionándole fiebre o "tiresa" $^{8}$ (cf. Vilca, 2009; Cladera, 2013), además de las consabidas lesiones en la piel. Al respecto, los niños reciben instrucciones concretas de parte de los adultos. Si no hay otra alternativa y tienen que detenerse en esos lugares, no solo deben realizar la challa de rigor sino que, además, no deben sentarse directamente sobre el suelo: es preciso que previamente hagan tres cruces sobre la tierra como una manera de pedir permiso a la Pachamama para hacerlo, o sentarse sobre una piedra, ${ }^{9}$ evitando el contacto directo. De otro modo, sería considerado una falta de respeto y podrían ser castigados por la tierra.

\section{Pisando fuerte}

Las sierras de Zenta y la serranía de Hornocal tienen una bien ganada reputación local de ser territorios muy difíciles de transitar. A la altitud de estas montañas y de los pasos que comunican espacialmente la quebrada del río Calete con los valles orientales se le suman la pendiente pronunciada del terreno y los caminos angostos bordeados por precipicios. Pero además, es preciso tener en cuenta que el suelo no es liso, sino que está tapizado de piedras; muchas de ellas, sueltas. No obstante, mediante el desarrollo cotidiano de una tactilidad familiarizada con este tipo de superficie (Taussig, 1995), el caminante va adquiriendo progresivamente un conocimiento corporal que le permite desplazarse en este ambiente sin problemas. Este radica fundamentalmente en reconocer perceptualmente las diferentes texturas del suelo con base en el grado y el tipo de fricción causada durante el movimiento de caminar entre el pie y el material sobre el que este apoya. De este modo, es capaz de ir encontrando durante la marcha una sucesión de puntos de apoyo estables que le permiten conservar el equilibrio en cada paso (cf. Vergunst, 2008). Esta manera de caminar es denominada en la zona como "pisar fuerte", y es una de las primeras habilidades que se deben adquirir para transitar esta topografía sin problemas. Cuando el terreno es particularmente pedregoso o luego de una nevada, algunos incluso envuelven sus tobillos con vendas para aumentar la estabilidad de la articulación y evitar una potencial caída. En los meses de invierno también vendan sus rodillas para evitar, además, la rigidez de las articulaciones ocasionada por el frío, la cual aumentaría la posibilidad de sufrir el desgarro de un músculo o de un tendón ante un resbalón.

Las pausas de descanso le permiten al viajero reponerse de este esfuerzo físico, confirmar su orientación en relación con ciertos rasgos del paisaje y evaluar su propio ritmo de marcha. Si se camina en compañía de otros, son momentos en los que se comparte alguna comida o bebida que se lleva en la mochila, o en los que se convida con hojas de coca para renovar el "acullico" ${ }^{10}$ que estuvieron mascando continuamente durante el trayecto. El coqueo es una práctica habitual en los Andes. Por un lado, los caminantes valoran las propiedades 
físicas de esta planta que alivia el cansancio, el frío y el hambre y los protege contra el apunamiento ocasionado por la hipoxia. Por otro, compartir la coca es un modo de interacción social; un gesto mediante el cual se expresa la solidaridad, la reciprocidad y la unión entre los miembros del grupo (cf. Bastien, 1978; Allen, 1981).

Pero aunque se trate de una ruta conocida y el caminante tenga sus propias técnicas y estrategias para transitarla, cada viaje y los riesgos que este conlleva son diferentes, ya que dependen de las condiciones del tiempo, de la eventual desaparición de trechos del sendero (ocasionados por deslizamientos del suelo), así como de las condiciones físicas y el estado de ánimo del viajero. Es por esto que el camino, aunque familiar, debe ser explorado y aprendido nuevamente en cada viaje y con cada paso (Vergunst, 2008). En un paisaje como el descripto, viajar con lluvia, apartarse mínimamente del sendero conocido, una distracción o el cansancio acumulado durante la marcha pueden derivar en un accidente, desde un simple tropezón hasta una caída grave. En ambos casos, se trata de eventos inesperados que no solo pueden ocasionar daños físicos, sino que además producen en el caminante un impacto emocional que influye en el modo en el que continúa la marcha. Es por esto que habitualmente este evalúa cuál es el mejor momento para emprender el viaje (época del año, condiciones del tiempo, hora del día) y realiza los preparativos adecuados para hacerlo con éxito.

Sin embargo, existe un grupo de caminantes que debe estar dispuesto a hacerlo en cualquier circunstancia y, a veces, sin tener demasiado conocimiento previo del paisaje: los agentes sanitarios. Ya sea para cumplir con sus rondas ${ }^{11} \mathrm{o}$ cuando son llamados para atender una emergencia, "salen" a los cerros cargando su mochila con medicamentos y, en algunas ocasiones, una carpa y suficiente comida y agua, elementos que les permiten completar recorridos de hasta dos días. ${ }^{12}$ Algunos llevan también un palo a modo de bastón sobre el que se van apoyando para hacer más liviana la marcha.

Las dificultades del terreno, el esfuerzo que requiere transitarlo con una carga como la descripta y el intenso ritmo de marcha que deben mantener para atender cuanto antes una urgencia médica muchas veces conducen al agotamiento físico de los agentes. Como consecuencia, a medida que aumenta su cansancio, el caminante comienza a percibir la textura del paisaje como una serie de obstáculos que se interponen a su paso (Van den Berg, 1952). En cada tropiezo, busca restablecer rápidamente el equilibrio, ya sea cambiando un pie de lugar o trasladando el peso de su cuerpo en la dirección opuesta a la que fue propulsado. Al mismo tiempo, "la vulnerabilidad de su cuerpo se le hace evidente, mucho tiempo antes de que caiga, en la pendiente peligrosa o en los objetos que se mueven bajo sus pies"13 (Van den Berg, 1952: 170). Pero si pese a esos ajustes no lo logra, la caída es inevitable. La postura erguida - mediante la cual experimenta el espacio - se pierde, y con ella, la relación habitual de su cuerpo con los elementos del ambiente. Al mismo tiempo, se desvanece la sensación de seguridad que le da el contacto con el suelo, lo cual genera desconcierto y un profundo temor. Siguiendo a Jackson, se trata de "una pérdida de equilibrio tanto físico como intelectual, una perturbación en el mismo centro y fundamento de nuestro ser" (Jackson, 1983: 329). ${ }^{14}$

Una vez producido el accidente, el viajero debe poner en práctica todas sus habilidades físicas para salir del problema. En ese proceso, todo su cuerpo es absorbido por los movimientos que realiza en estrecha relación con el paisaje (Van den Berg, 1952) con el fin de detener la caída y volver al camino seguro:
11. Recorridos para visitar las casas de las familias que les fueron asignadas para su control.

12. Es importante destacar que, en la zona de estudio, este tipo de trabajo es realizado tanto por varones como por mujeres.

13. Mi traducción.

14. Mi traducción. 
15. Empinado.

16. En los niños, el "susto" viene acompañado además por fiebre y diarrea, síntomas que son menos frecuentes en el adulto.

17. Siguiendo a Costilla (20082010), se puede hablar de una cosmovisión religiosa que sintetiza dialécticamente elementos del cristianismo y de la religiosidad local prehispánica. "cavaba con las manos y con los pies, primero una, así, y [después] la otra, porque ¡ahí te funciona todo! Pero me las ingenié y subí" (Marcela, 34 años, agente sanitaria). Sin embargo, antes de continuar la marcha, el caminante debe además reponerse anímicamente. En ese sentido, es preciso recordar que, debido a las características de los caminos por los que se transita, en esta zona una caída es frecuentemente sinónimo de muerte. Por lo tanto, estos episodios tienen un fuerte impacto en la subjetividad del caminante y constituyen hitos que marcan la experiencia del viaje: "Tenés que tener mucho coraje, porque es tan parado ${ }^{15}$ así el cerro que vos decís: 'te caés y no le contás a nadie'. Y bueno, tuve que agarrar coraje y salir después del susto" (Nancy, 35 años, agente sanitaria). Este "susto" tiene además otras implicancias para el viajero ya que su "ánimo" (o fuerza vital) puede salir de su cuerpo. Como consecuencia, la persona se siente débil, decaída, duerme intranquila y se despierta sobresaltada. ${ }^{16} \mathrm{El}$ "asustarse" genera, a su vez, un vínculo con el lugar en el que la persona perdió su entidad anímica al que, en ciertos casos, debe volverse para recuperarla y curarse. Es por esto que cuando se inicia una caminata se dice que hay que ir con "coraje", es decir, con la fortaleza espiritual necesaria para evitar que el cerro lo "agarre" (cf. Bugallo y Vilca, 2011).

Pero más allá de la seguridad que pueda tener sobre sus propias fortalezas y habilidades, el caminante de los cerros confía en la ayuda y protección que le puedan brindar diferentes seres tutelares. Como ya fue mencionado, todos los caminantes solicitan protección a la Pachamama para el viaje. No obstante, dentro del sistema religioso propio de esta región (Merlino y Rabey, 1992; Costilla, 2008-2010), ${ }_{17}$ cuando ocurre este tipo de accidentes también se invoca la ayuda de santos del panteón católico vinculados de manera directa con el poder de Dios. En ese sentido, el hallazgo de un objeto (como una piedra o una rama de la cual sujetarse) o la ocurrencia de un hecho particular que le haya permitido salir airoso del incidente (como la invocación al santo que coincide con el celebrado en el calendario litúrgico) son interpretados como la respuesta a la intervención divina solicitada. El relato de Bárbara, otra de las agentes entrevistadas que trabajó en la zona de Caspalá, es esclarecedor en este sentido:

Caí y quedé colgada y miraba para abajo, y el río era un hilito, un hilito... entonces yo digo “¿cómo hago?”. Rezaba nada más, rezaba... yo lo único que dije ese día (era el día de San José) [fue] “iAy, San José, por favor!, ique no me pase nada!”. Y lo único que hice es gritar y decía “iJesús, Jesús, Jesús!”... Entonces yo decía eso, y estiro el brazo y con la mano izquierda me agarro de una piedra. Y cuando yo levanto la vista, “¿estoy agarrada de una piedra?” digo yo. Porque yo gracias a una piedra me caí, y gracias a otra piedra me salvé (Bárbara, 33 años, agente sanitaria).

\section{Entre el cielo y la tierra}

De acuerdo con lo analizado hasta aquí, la experiencia de caminar implica una interacción activa entre el caminante y el medio ambiente por el que se desplaza. Por lo tanto, el tiempo atmosférico, en tanto parte dinámica de ese medio, también interviene en la coproducción de dicha experiencia (Ingold y Vergunst, 2005).

La Quebrada de Humahuaca presenta un clima árido con inviernos fríos y secos, y con una concentración de lluvias en los meses de verano. Aunque la llegada de estas es deseada por todos para que prosperen los cultivos, también producen daños y aumentan considerablemente los riesgos al momento de 
viajar a pie. Por un lado, las precipitaciones en la cuenca alta de los ríos dan lugar a crecientes grandes y violentas, que erosionan los terrenos ubicados en sus márgenes y producen aludes o "volcanes" en las quebradas subsidiarias. Por otro, si bien son de corta duración, tienen gran intensidad y se acompañan de frecuentes caídas de rayos. Estos suelen ocasionar la muerte instantánea de muchos caminantes durante la época de verano, en especial cuando transitan por espacios abiertos, por lugares elevados o por suelos que contienen una gran cantidad de minerales en su estructura, como por ejemplo, los médanos.

Son pocos los recursos prácticos que tienen los viajeros para defenderse de los rayos durante la marcha: los pobladores de esta zona han aprendido de sus padres que deben quedarse quietos y, sobre todo, "no meterse bajo las plantas", porque aumenta el peligro de ser alcanzados por una descarga. Del mismo modo, se alejan de los rebaños de animales, ya que consideran que el pelaje puede atraer la electricidad del rayo, estableciendo una asociación entre esta y la estática que se produce al frotar la lana. También se alejan de las víboras, aunque en este caso la asociación reside en la similitud del movimiento del animal con el trayecto que hace el rayo en el cielo. ${ }^{18}$ Las radios o cualquier otro objeto metálico que lleven consigo son arrojados rápidamente para evitar que atraigan la descarga hacia el caminante.

Pero en los Andes, estos fenómenos meteorológicos no son solo manifestaciones de un tipo determinado de clima. Las lluvias, las nubes, la neblina y el rayo son también considerados fenómenos de transición relacionados con las chakanas ${ }^{19}$ y puentes de correspondencia entre dos niveles cósmicos: el cielo y la tierra. Por su precariedad y peligrosidad merecen una dedicación ritual por parte de las personas para mantener el flujo adecuado de las relaciones entre ambos niveles (Estermann, 2006). Cuando este flujo es interrumpido, en particular por la falta del hombre en establecer ética y ceremonialmente dichas relaciones, "la muerte vence sobre la vida" (Estermann 2006: 184). Por consiguiente, además de sus habilidades prácticas para transitar el paisaje en medio de una lluvia, el caminante debe conocer también ciertas estrategias y gestos rituales que le permitan conjurar la aparición y evitar las eventuales consecuencias de estos fenómenos durante el viaje.

Al igual que ocurre con otros seres de la cosmología andina, los poderes del rayo son ambiguos: por un lado, puede ocasionar muerte, destrucción, sequías, o producir lo doble y lo monstruoso (cf. Rösing, 1996; Rivière, 1997; van Kessel, 2003; Bugallo, 2009). Por otro, puede otorgar poderes y capacidades de curación, ya sea a personas (Rösing, 1996) o a elementos del paisaje como las piedras (Bugallo, 2009; Vilca, 2009). Pero además, dentro del sistema religioso andino, el rayo es vinculado también con santos del panteón católico. Si bien en otras regiones se lo asocia de manera preponderante con san Santiago (Fernández Juárez, 1995; Rivière, 1997; Bugallo, 2009), en el área en que se realizó este estudio, esta asociación se hace con santa Bárbara. Esta es una figura relacionada con las tormentas, los rayos y la lluvia como principio de la productividad y de la fertilidad general (Gisbert, 1980; Gade 1983). Por una parte, los pobladores le solicitan agua para los cultivos y los animales en épocas de sequía. Por otra, todos los caminantes solicitan su protección cuando los sorprende una tormenta durante la marcha. Los modos de hacerlo son transmitidos generacionalmente en las familias como una parte más del conocimiento que se considera necesario poseer para transitar en el paisaje. Para algunos solo es preciso repetir varias veces su nombre: "Mi papá, mi mamá, mi abuela me decían que tenemos que invocar a santa Bárbara. Así al rayo, decimos 'Santa Bárbara, Santa Bárbara, Santa Bárbara' para que no nos agarre en medio de la tormenta. Tenés que repetirlo"
18. En algunas regiones de los Andes, las serpientes constituyen la representación de la antigua divinidad del rayo/relámpago, y participan de rituales realizados para interrumpir las lluvias excesivas (cf. Rivière, 1997).

19. Según Estermann (2006), chakana es un vocablo quechua que proviene del verbo chakay, que significa "cruzar" más el sufijo -na que lo convierte en sustantivo. Chakana es entonces el "cruce" entre dos puntos. En ese sentido, “La chakana [...] es el "punto de transición' entre el arriba/abajo y derecha/izquierda; es prácticamente el símbolo andino de la relacionalidad del todo. [...Es] además el elemento de conexión [...] entre los principios de correspondencia (vertical) y complementariedad (horizontal)" (Estermann 2006: 170-171). El eje vertical indica la relacionalidad entre el micro y el macrocosmos; y el eje horizontal, entre lo femenino y lo masculino. 
(Elías, 31 años, residente de Ocumazo). Otros refuerzan la invocación a la santa con un rezo: “digo 'Santa Bárbara' y ¡te rezás el rezo que sabés, pues!” (Doña Mercedina, 75 años, residente de Ocumazo). Finalmente otros caminantes agregan la protección extra que les puede brindar el ángel de la guarda, la cual es representada por un rosario negro o azul que llevan colgado en el cuello. Debido a que su valor como elemento protector es amplio ("contra todo mal", de acuerdo con los entrevistados), también suele ser utilizado para alejar las tormentas.

\section{Conclusiones}

En este trabajo se realizó una exploración etnográfica de la práctica de caminar entre los pobladores de un sector rural de la Quebrada de Humahuaca, en el marco de sus actividades cotidianas y también en situaciones concretas de infortunio, con el fin de analizar diversos aspectos del conocimiento que utilizan para transitar por el paisaje.

Dicho conocimiento surge a partir de la experiencia corporal basada en la integración subjetiva y dinámica del caminante con los diferentes elementos del paisaje por el que transita. Mediante un aprendizaje basado en la percepción, los pobladores del área de estudio saben cómo desplazarse en el terreno, encontrar el camino y detectar o reponerse de eventuales situaciones de peligro durante la marcha. En ese sentido, se puede hablar del desarrollo de una "inteligencia" corporal total, ya que uno no camina solo con las piernas y los pies, sino que lo hace con todo el cuerpo. Las pendientes, las diferentes superficies que se van pisando y los eventuales tropiezos o caídas en el trayecto obligan a reajustes continuos de los movimientos del cuerpo durante la marcha. Del mismo modo, el caminante no percibe el ambiente por el que se mueve usando solamente el sentido de la vista y, eventualmente, la audición. Le es preciso activar múltiples sentidos, algunos de los cuales - como el equilibrio, el tacto, la kinestesia y la propiocepción - suelen pasar desapercibidos cuando se camina por una superficie plana y lisa.

En la zona de estudio, además, este conocimiento práctico lleva inserta la vivencia de lo poderoso y los modos de vincularse con diversas alteridades que forman parte de la multidimensionalidad del paisaje andino, modos que se encuentran imbricados en la misma práctica de caminar. Mediante diversos gestos rituales (challas, pedidos de permiso, trazado de cruces en el suelo), el caminante se relaciona y cumple con los deberes de reciprocidad hacia la tierra sobre la que transita. Asimismo, invoca la intercesión y protección de figuras del panteón católico para enfrentarse al peligro que encierran las tormentas y los rayos como fenómenos intermediarios entre el orden cósmico y el orden humano, o ante el eventual riesgo de muerte en una caída.

\section{Agradecimientos}

A todos los caminantes de la Quebrada que compartieron su tiempo y sus experiencias conmigo. Al Dr. César Ceriani Cernadas por sus valiosos comentarios, que han contribuido a mejorar este artículo.

A Justina, por sus enseñanzas y su guía para realizar esta investigación. 


\section{Q Bibliografía}

》 ABELEDO, Sebastián. 2010. "Los viajes de intercambio en la Puna de Atacama: pasado reciente y actualidad del caravaneo en Santa Rosa de los Pastos Grandes (Salta)". VIo Congreso de Ciudades y Pueblos del Interior. Catamarca.

» ABERCROMBIE, Thomas. 1998. Pathways of Memory and Power. Ethnography and History among an Andean People. Madison, Londres: The University of Wisconsin Press.

» ALBÓ, Xavier. 1991. “La experiencia religiosa aymara”. En: M. Marzal, R. Robles, E. Maurer, X. Albó y B. Melía. Rostros indios de Dios. Los amerindios cristianos. Quito: Abya Yala. pp. 201-265.

》 ALLEN, Catherine. 1981. "To be Quechua: The Symbolism of Coca Chewing in Highland Peru”. American Ethnologist, 8 (1): 157-171.

" ANDERSON, Jeanine. 2013. "Movimiento, movilidad y migración: una visión dinámica de la niñez andina". Bulletin de l'Institut Français d' Études Andines, 42 (3): 453-471.

» ARZENO, Mariana. 2011. "El campesinado de la Quebrada de Humahuaca. Análisis de su transformación desde un enfoque geográfico”. En: M. Kollman (Coord.). Espacio, espacialidad y multidisciplinariedad. Buenos Aires: Eudeba. pp. 177-208.

» BASTIEN, Joseph. 1978. Mountain of the Condor. Metaphor and ritual in an Andean ayllu. Minnesota: West Publishing.

» BUGALLO, Lucila. 2009. "Quipildores: marcas del rayo en el espacio de la Puna jujeña”. Cuadernos FHyCS- UNJu, 36: 177-202.

» BUGALLO, Lucila. 2016. “Wak'as en la puna jujeña. Lo fluido y lo fino en el diálogo con la Pachamama". En: L. Bugallo y M. Vilca (Comps.). Wak'as, diablos y muertos. Alteridades significantes en el mundo andino. San Salvador de Jujuy: Editorial de la Universidad Nacional de Jujuy-EDIUNJU, Instituto Francés de Estudios Andinos. pp. 111-161.

" BUGALLO, Lucila y MAMANI, Lina. 2014. "Molinos en la Quebrada de Humahuaca: lugares de encuentro de gentes y caminos. La región molinera del norte jujeño, 19401980". En: A. Benedetti y J. Tomasi (Comps.). Espacialidades altoandinas. Nuevos aportes desde la Argentina: Miradas hacia lo local, lo comunitario y lo doméstico. Ciudad Autónoma de Buenos Aires: Editorial de la Facultad de Filosofía y Letras Universidad de Buenos Aires. pp. 63-118.

» BUGALLO, Lucila y TOMASI, Jorge. 2012. "Crianzas mutuas. El trato a los animales desde las concepciones de los pastores puneños (Jujuy, Argentina)". Revista Española de Antropología Americana, 42 (1): 205-224.

» BUGALLO, Lucila y VILCA, Mario. 2011. "Cuidando el ánimu: salud y enfermedad en el mundo andino (Puna y Quebrada de Jujuy, Argentina)". Nuevo Mundo Mundos Nuevos. [En linea]. En http://nuevomundo.revues.org/61781. Fecha de consulta: 06 julio 2017. DOI: https://doi.org/10.40oo/nuevomundo.61781. Fecha de acceso: 06/7/2017.

" CLADERA, Jorge. 2010. “Continuidad y cambio en las prácticas trashumantes. El caso de los ganaderos del circuito Iscuya-Astilleros, comunidad Kolla. Finca Santiago (provincia de Salta)". Kula. Antropólogos del Atlántico Sur, 3: 55-65.

" CLADERA, Jorge. 2013. “Pessoas que Cruzam Territórios e Territórios que sao Pessoas -As Experiências do Direito Espacial nos Andes: um estudo de caso no noroeste argentino". Ilha, 15 (1): 149-178. 
»COSTILLA, Julia. 2008-2010. “Cristianismo indígena en el NOA: resignificaciones y resimbolizaciones en relatos orales del siglo XX (1940-1998)". Cuadernos del Instituto Nacional de Antropología y Pensamiento Latinoamericano, 22: 59-70.

»CRUZ, Pablo. 2012. "El mundo se explica al andar. Consideraciones en torno a la sacralización del paisaje en los Andes del sur de Bolivia (Potosí, Chuquisaca)”. Indiana, 29: 221-251.

»ELIZAGA, Julieta. 2010. Uywa ñan, runa ñan: caminos de rebaños, caminos de personas. Una aproximación a los modos de conocer en un contexto de pastores de altura en los Andes meridionales. Tesis Doctoral, Universidad de Tarapacá.

" ESTERMANN, Josef. 2006. Filosofía andina. Sabiduría indígena para un mundo nuevo. La Paz: ISEAT.

» FERNÁNDEZ GARCíA, Javier, TECCHI, Rodolfo, RABEY, Mario. 1992. “La ganadería de Ilamas en el altiplano jujeño”. En: A. Veloso y R. A. Tecchi (Comps.). Ecosistemas altoandinos de Argentina y Chile. Memorias del Programa de Ecología Regional, 3. San Salvador de Jujuy: Universidad Nacional de Jujuy. pp. 65-68.

» FERNÁNDEZ JUÁREZ, Gerardo. 1995. El banquete aymara. Mesas y yatiris. Colección “Biblioteca Andina". La Paz: Editorial Hisbol.

" GADE, Daniel. 1983. "Lightning in the Folklore and Religion of the Central Andes". Anthropos, 78: 770-788.

» GASKINS, Suzanne y PARADISE, Ruth. 2010. "Learning through observation in daily life”. En D. Lancy, J. Bock y S. Gaskins (Eds.). The Anthropology of Learning in Childhood. Plymouth: Altamira Press. pp. 85-117.

» GIBSON, James. 1979. The ecological approach to visual perception. Boston: Houghton Mifflin.

» GIL GARCÍA, Francisco y FERNÁNDEZ JUÁREZ, Gerardo. 2008. “El culto a los cerros en el mundo andino: estudios de caso". Revista Española de Antropología Americana, 1: 105-113.

» GISBERT, Teresa. 1980. Iconografía y mitos indígenas en el arte. La Paz: Gisbert.

» GÖBEL, Bárbara. 1998. "Salir de viaje: Producción pastoril e intercambio económico en el noroeste argentino”. En: S. Dedenbach-Salazar, C. Arellano, E. König y H. Prümers (Eds.). 50 años de Estudios americanistas en la Universidad de Bonn. Nuevas contribuciones a la arqueología, etnohistoria, etnolingüística y etnografía en las Américas. Saurwein: Estudios Americanistas de Bonn. pp. 867-891.

» GÖBEL, Bárbara. 2000-2002. “Identidades sociales y medio ambiente: la multiplicidad de los significados del espacio en la Puna de Atacama”. Cuadernos del Instituto Nacional de Antropología y Pensamiento Latinoamericano, 19: 267-296.

" GÖBEL, Bárbara. 2002. "La arquitectura del pastoreo: Uso del espacio y sistemas de asentamientos en la Puna de Atacama (Susques)". Estudios Atacameños, 23: 53-76.

» GRILLO FERNÁNDEZ, Eduardo. 1994. “El paisaje en la cultura andina de siempre”. En: E. Grillo, V. Quiso, G. Rengifo y J. Valladolid (Eds.). Crianza andina de la chacra. PRATEC. http://www.pratecnet.org/pdfs/Crianza\%20andina\%20de\%2ola\%20chacra.pdf. pp. 1345 .

» INGOLD, Tim. 2000. The Perception of the Environment. Essays in Livelihood, Dwelling and Skill. Londres y Nueva York: Routledge.

» INGOLD, Tim. 2008. “Tres en uno: Cómo disolver las distinciones entre cuerpo, mente y cultura”. En: T. Sánchez-Criado (Coord.). Tecnogénesis. La construcción técnica de las ecologías humanas, Vol. 2. Madrid: AIBR. pp. 1-33. 
"INGOLD, Tim. 2011. Being Alive. Essays on Movement, knowledge and description. Londres: Routledge.

»INGOLD, Tim y VERGUNST, Jo Lee. 2005. "The eye of the storm: visual perception and the weather". Visual Studies, 20 (2): 97-104.

»ISBELL, Billie Jean. 1978. To defend ourselves: ecology and ritual in an Andean village. Austin: University of Texas, Institute of Latin American Studies.

" JACKSON, Michael. 1983. "Knowledge of the body”. Man, New Series, 18 (2): 327-345.

" JACKSON, Michael. 1989. Path toward a clearing. Radical empiricism and ethnographic inquiry. Bloomington: Indiana University Press.

" JACKSON, Michael (Ed.). 1996. "Introduction: Phenomenology, Radical Empiricism and Anthropological Critique". En: Things as they are. New Directions in Phenomenological Anthropology. Bloomington e Indianápolis: Indiana University Press. pp. 1-50.

" KARASIK, Gabriela. 1984. “Intercambio tradicional en la Puna jujeña”. Runa, 14: 56-78.

»LEMA, Verónica. 2014. “Criar y ser criados por las plantas y sus espacios en los Andes Septentrionales de la Argentina". En: A. Benedetti y J. Tomasi (Comps.). Espacialidades altoandinas. Nuevos aportes desde la Argentina: Miradas hacia lo local, lo comunitario y lo doméstico. Ciudad Autónoma de Buenos Aires: Editorial de la Facultad de Filosofía y Letras Universidad de Buenos Aires. pp. 301-338.

»LEMA, Verónica y PAZZARELLI, Francisco. 2015. "Memoria fértil. Crianza de la historia en Huachichocana". Nuevo Mundo Mundos Nuevos [En ligne]. En: http://journals.openedition.org/nuevomundo/67976. DOI: https://doi.org/10.40oo/nuevomundo.67976. Fecha de acceso: 18/7/2018.

" MARTínEZ, Gabriel. 1969. “Los dioses de los cerros en los Andes”. Journal de la Société des Américanistes, LXIX: 85-115.

" MARTínEZ, Gabriel. 1989. Espacio y Pensamiento. La Paz: Hisbol.

" MARTíNEZ, Bárbara. 2014. “Cartografías en tránsito: Mapas orales y memoria social en El Cajón (Catamarca, Argentina)”. Runa, 35: 77-92.

» MAYER, Enrique. 1974. “Las reglas del juego en la reciprocidad andina”. En: G. Alberti y E. Mayer (Comps.). Reciprocidad e intercambio en los Andes peruanos. Lima: IEP Ediciones. pp. 37-65.

" MERLINO, Rodolfo y RABEY, Mario. 1983. "Pastores de altiplano andino meridional: religiosidad, territorio y equilibrio ecológico". Allpanchis, 21: 149-171.

" MERLINO, Rodolfo y RABEY, Mario. 1992. "Resistencia y hegemonía: cultos locales y religión centralizada en los Andes del sur”. Allpanchis, 40: 173-200.

» NIELSEN, Axel. 2003. "Por las rutas del Zenta: Evidencias directas de tráfico prehispánico entre Humahuaca y las Yungas". En: G. Ortiz y B. Ventura (Eds.). La mitad verde del mundo andino: investigaciones arqueológicas en la vertiente oriental de los Andes y las Tierras Bajas de Bolivia y Argentina. San Salvador de Jujuy: Editorial de la Universidad Nacional de Jujuy. pp. 261-284.

»ONG, Walter. 2006. Oralidad y escritura. Tecnologías de la palabra. Buenos Aires: Fondo de Cultura Económica.

» ORTIZ RESCANIERE, Alejandro. 1989. "La comunidad, el parentesco y los patrones de crianza andinos". http://ezproxybib.pucp.edu.pe/index.php/anthropologica/article/ viewFile/2067/1998.

" PARADISE, Ruth y ROGOFF, Barbara. 2009. "Side by Side: Learning by Observing and Pitching In". Ethos. Journal of the Society for Psychological Anthropology, 37 (1): 102-138. 
»PARDO, Elena. 1997. “Crianza de los niños según la concepción andina”. En: J. van Kessel y H. Larraín Barrios (Eds.). Manos sabias para criar la vida. Tecnología andina. Aportes al Simposio: Tecnología Tradicional Andina Actual del 49 Congreso Internacional de Americanistas. Quito: Ecuador. pp. 145-152.

»RABEY, Mario y MERLINO, Rodolfo. 1988. “El control ritual-rebaño entre los pastores del sur de los Andes centrales (Argentina). En: J. Flores Ochoa (Ed.). Llamichos y paqocheros: Pastores de llamas y alpacas. Cuzco: Consejo Nacional de Ciencia y Tecnología. pp. 113-120.

» RABEY, Mario, MERLINO, Rodolfo, GONZÁLEZ, Daniel. 1986. “Trueque, articulación económica y racionalidad campesina en el sur de los Andes Centrales”. Revista Andina, 4 (1): 131-16o.

» RIVIÈRE, Gilles. 1997. “Tiempo, poder y sociedad en las comunidades Aymaras del Altiplano (Bolivia)”. En: M. Goloubinoff, E. Katz y A. Lammel (Eds.). Antropología del Clima en el mundo Hispanoamericano. Tomo II. Quito: Abya Yala. pp. 31-54.

»RÖSING, Ina. 1996. El rayo. Amenaza y vocación. Creencia y ritual en los Andes Bolivianos. Ulm: Ulmer Kulturanthropologische Schriften, Band 8.

»SENDÓN, Pablo. 2010. “La tierra emparentada. Acerca de los muyu o "suertes” (sistema de barbecho sectorial) en Marcapata, Perú”. Estudios Atacameños. Arqueología y Antropología Surandinas, 40: 63-84.

» TAUSSIG, Michael. 1995. Un gigante en convulsiones. El mundo humano como sistema nervioso en emergencia permanente. Buenos Aires: Gedisa.

» TOMASI, Jorge. 2013. Espacialidades pastoriles en las tierras altoandinas. Asentamientos y movilidades en Susques, puna de Atacama (Jujuy, Argentina). Revista de Geografía Norte Grande, 55: 67-87.

» TOMASI, Jorge. 2014. “De los pastoreos a la casa. Espacialidades y arquitecturas domésticas entre los pastores altoandinos (Susques, provincia de Jujuy)”. En: A. Benedetti y J. Tomasi (Comps.). Espacialidades altoandinas. Nuevos aportes desde la Argentina: Miradas hacia lo local, lo comunitario y lo doméstico. Ciudad Autónoma de Buenos Aires: Editorial de la Facultad de Filosofía y Letras Universidad de Buenos Aires. pp. 257-299.

» TURNER, Victor. 1982. From ritual to theatre. Nueva York: Performing Arts Journal Press.

» TURNER, Victor. 1985. On the Edge of the Bush. Anthropology as Experience. Tucson: University of Arizona Press.

» TURNER, Victor y BRUNER, Edward. 1986. The Anthropology of Experience. Urbana y Chicago: University of Illinois Press.

»VAN DEN BERG, Jan. 1952. "The Human Body and the Significance of Human Movement: A Phenomenological Study”. Philosophy and Phenomenological Research, 13 (2): 159-183.

»VAN KESSEL, Juan. 1992. Aica y la Peña sagrada. Iquique: El jote errante y Puno, CIDSA.

» VAN KESSEL, Juan. 2003. “Individuo y religión en los Andes”. Cuadernos de investigación en Cultura y Tecnología Andina, № 16. http://www.iecta.cl/biblioteca/cuadernos/pdf/cuaderno_16.pdf. Fecha de acceso: 19/7/2018.

» VERGUNST, Jo Lee. 2008. “Taking a Trip and Taking care in Everyday Life”. En: T. Ingold y J. Vergunst (Eds.). Ways of Walking. Ethnography and Practice on Foot. Aberdeen: Ashgate. pp. $105-121$.

» VILCA, Mario. 2009. “Más allá del “paisaje”. El espacio de la Puna y la Quebrada de Jujuy: ¿comensal, anfitrión, interlocutor?”. Cuadernos FHyCS-UNJu, 36: $245-259$. 